\title{
Diversity, Medicinal Uses and Conservation Status of Medicinal Plants at Mandaragiri, Angul Forest Division, Odisha, India
}

\author{
Rudra Narayan Pradhan ${ }^{1, *}$, Omprakash Rautaraya ${ }^{2}$, Prasanna Behera ${ }^{3}$, Sujogya Kumar Panda ${ }^{4}$ \\ ${ }^{1}$ Regional Chief Conservator of Forests, Angul, Odisha, India \\ ${ }^{2}$ Freelance Consultancy, Pl.No-537 GA colony, Bharatpur, Bhubaneswar, India \\ ${ }^{3}$ Nature Environment \& Wildlife Society (NEWS) Angul, Odisha, India \\ ${ }^{4}$ Dept. of Zoology, North Orissa University, Baripada -757003, India \\ *Corresponding Author: rudra_pradhan_nou@yahoo.co.in
}

Copyright $(\underset{0}{2014}$ Horizon Research Publishing All rights reserved.

\begin{abstract}
The present paper provides the status of medicinal plant species growing in and around the tribal settlements of Mandaragiri region and emphasizes on their medicinal uses. A total number of eighty two species belonging to seventy four genera and forty families are represented as medicinal value. Species such as Acacia catechu, Acacia nilotica, Buchanania lanzan, Emblica officinalis, Gardenia sp., Indigofera cassioides, Shorea robusta and Woodfordia fruticosa are rare at Mandaragiri forest. Whereas, plants like Rauvolfia serpentina, Santalum album, Streblus asper and Spondias pinnata are endangered species at Mandaragiri forest.
\end{abstract}

Keywords Medicinal Plants, Rare, Endangered

\section{Introduction}

Plants are the basis of life on earth, supplying fresh oxygen and play an important role to people's livelihood. Worldwide, 422,000 seed plants are reported and more than 50,000 of them are used for medicinal purposes [1]. Besides, $85 \%$ of traditional medicine practices used in primary healthcare is derived from plants. India and China, two of the largest Asian countries, have the richest arrays of registered and relatively well known medicinal plants [2]. India is one of the twelve mega biodiversity countries of the world having rich vegetation with a various medicinal value. About $43 \%$ of plants from Indian subcontinent (approximately 7,500 species) are reported to have medicinal value [3]. Indian subcontinent is being inhabited by over 53.8 million tribal people in around 5,000 villages with 427 tribal communities dominated by forest. Among the states of India, Odisha has dense forests with natural plant resources. The tribes residing deep inside the forest are still depending on medicinal plants for their primary health care and treatment of diseases. Odisha state has 62 categories of tribes. These ethnic communities have good acquired knowledge on medicinal uses of plants in the course of their lifetime. The rural people of the state depend on the traditional ethno-medicine for their day-to-day primary health care. These medicinal plants gain further importance in the regions where modern medical health facilities are either not available or not easily accessible.

The dialectical relationship between indigenous knowledge and practices shapes the ecosystem and affects the constituent plant populations [4]. By incorporating indigenous knowledge and use in the process of scientific research, new hypotheses for the sustainable conservation of the resources can be developed [5]. Indigenous knowledge and use have to be analyzed to develop appropriate management measures that build on both scientific and local knowledge [6]. Due to the changing perception of the local people, and the ever increasing influence of global commercialization and socio-economic transformation, indigenous knowledge on plant resource use is constantly diminishing [7, 8]. In addition to this lack of organized sustainable and scientifically monitored cultivation and harvesting management techniques, lack of awareness of social factors, the number of usefulness of plant resources is decreasing at an alarming rate [8]. The continuous exploitation of several wild medicinal plant species have resulted in population decline of many high value medicinal plants. Furthermore, the indigenous knowledge on the use of lesser known plants declines rapidly [9].

Until today, there are not any studies about the medicinal plants and their uses from the Mandaragiri region inhabitants. The present paper aims to record in detail the status of medicinal plant species growing in and around the tribal settlements of Mandaragiri region and to emphasize on their medicinal uses.

\section{Study Area}


The Mandaragiri forest lies between $20^{\circ} 47^{\prime} 15^{\prime \prime}$ and $20^{\circ}$ $49^{\prime} 00^{\prime \prime}$ north latitude and $85^{\circ} 11^{\prime} 30^{\prime \prime}$ and $85^{\circ} 3^{\prime} 30^{\prime \prime}$ ' east longitude in the district of Angul, Odisha, India. The Mandaragiri forest is a unique fragile ecosystem covering 586 hectares of forest land (Figures $1 \& 2$ ). The whole mountain was covered with dense forest but due to lack of protection, a rapid, large scale felling of trees occurred since 1970, diminishing the forest cover. In 1995, the people of the surrounding nine villages formed the Mandaragiri Surakshya Manch in order to protect the forest. Now the mountain boasts a thick sprawling green and many useful plants. Inhabitants around Mandaragiri hill are attached to it and have a basic natural feeling for its conservation due to different religious beliefs and mythological reasons. The climate of Mandaragiri is warm and humid. Summer months are very hot with maximum day temperatures $45-47^{\circ} \mathrm{C}$ in the months of May and June which drops sensibly with the onset of the monsoon. When the monsoon withdraws in October, the daytime temperature remains the same however the nights become cooler. Gradually, both day and night temperatures fall. The coolest month is December when temperature at night varies between $6^{\circ} \mathrm{C}$ and $11^{\circ} \mathrm{C}$. Relative humidity is maximum in October and minimum in April and May.

\section{Materials and Methods}

Field study (16 trip) was conducted from time to time during both winter and summer to generate the information's by contacting village medicine men and Kavirajs (Vaidyas). They were interviewed to record different plants used in primary healthcare system for various remedies. The spot identification of medicinal plants was done with the help of local experts and published floras [10]. Fresh samples of species which could not been identified were brought to the institution and identified in the laboratory. The diversity and place of distribution was recorded. Nativity and endemism were identified based on the distribution of the species.

To establish the uses of the medicinal plants literature was composed from the earlier published books by Kirtikar et al. [11-13]. In the following tabulation, the plant names have been arranged alphabetically accordingly their botanical names.

\section{Results}

A Total number of 82 species belonging to 74 genera and 40 families were recorded. Fabaceae $(9$ species $)$; Anacardiaceae (5); Mimosaceae (5); Moraceae (5); Apocynaceae (4); Caesalpiniaceae (4); Combretaceae (4) and Verbenaceae (4) are the dominated families. Ficus sp., Terminalia sp. and Acacia sp. are the dominated genera. Among the medicinal plants Anogeissus latifolia, Gmelina arborea, Madhuca longifolia, Pterocarpus marsupium, Shorea robusta, and Terminalia bellirica are high value species exploited for different purposes. Species such as Acacia catechu, Acacia nilotica, Buchanania lanzan, Emblica officinalis, Gardenia sp., Indigofera cassioides, Shorea robusta and Woodfordia fruticosa are rare at Mandaragiri forest. Whereas, plants like Rauvolfia serpentina, Santalum album, Streblus asper and Spondias pinnata are endangered species at Mandaragiri forest. (Table $1)$.

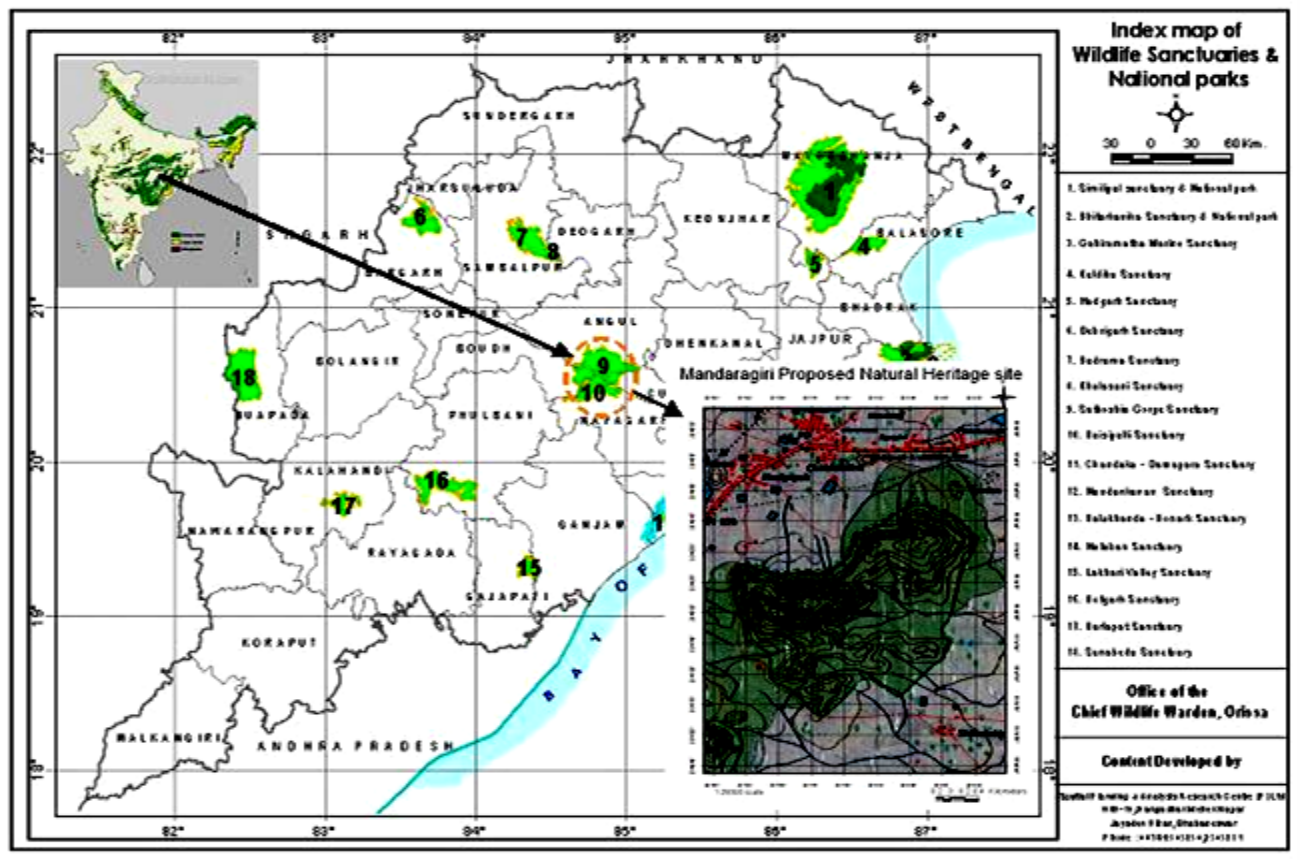

Figure 1. Map of OdishaIndia showing Mandaragiri forest 


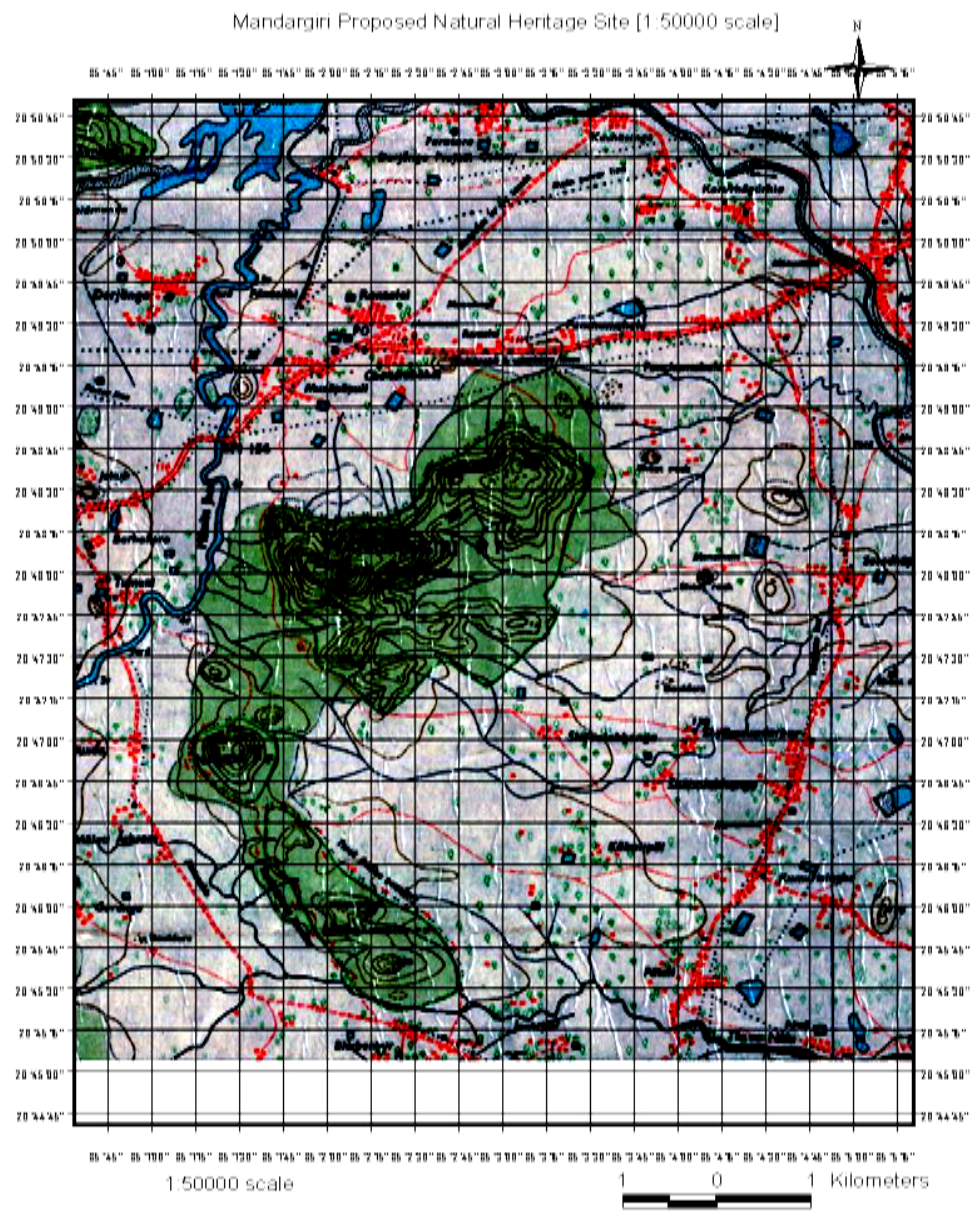

Figure 2. Map of the Mandaragiri forest

Table 1. Important ethno-medicinal plants of Mandaragiri forest

\begin{tabular}{|c|c|c|c|c|c|c|}
\hline Botanical Name & Family & Local Name & $\begin{array}{l}\text { Status of } \\
\text { Finding }\end{array}$ & $\begin{array}{c}\text { Place of } \\
\text { distribution }\end{array}$ & $\begin{array}{l}\text { Parts } \\
\text { Used }\end{array}$ & Medicinal Properties \\
\hline Abrus pracatorius $\mathrm{L}$. & Fabaceae & Kaincha & MQ & V & $\begin{array}{l}\text { Rt, Lf, } \\
\text { Sd. }\end{array}$ & $\begin{array}{c}\text { Leucoderma, skin diseases asthma and } \\
\text { fever. }\end{array}$ \\
\hline $\begin{array}{l}\text { Acacia nilotica }(1 .) \\
\text { Willd. ex Delile sp. } \\
\text { indica } \\
\end{array}$ & Mimosaceae & Babul & $\mathrm{CF}$ & F H & $\mathrm{Bk}$, gum & $\begin{array}{l}\text { Haemostatic, antipyretic, asthma, } \\
\text { diarrhea }\end{array}$ \\
\hline Acacia catechu & Mimosaceae & Khair & MQ & E & $\mathrm{Bk}, \mathrm{Hw}$ & $\begin{array}{c}\text { Anti-dysenteric, haemostatic, leprosy, } \\
\text { leucoderma }\end{array}$ \\
\hline $\begin{array}{l}\text { Acacia leucophloea } \\
\text { (Roxb.) Willd. }\end{array}$ & Mimosaceae & Gohira & $\mathrm{R}$ & F H & $\mathrm{Bk}$ & $\begin{array}{c}\text { Thermogenic, constipating, antipyretic, } \\
\text { used in cough, bronchitis, leprosy, } \\
\text { vomiting and ulcers }\end{array}$ \\
\hline $\begin{array}{l}\text { Achyranthes aspera } \\
\text { L. }\end{array}$ & Amaranthaceae & Apama-ranga & $\mathrm{CF}$ & $\mathrm{E}$ & Wp & $\begin{array}{l}\text { Useful in cough, asthma, bronchitis, colic } \\
\text { and cardiac disorder. }\end{array}$ \\
\hline $\begin{array}{l}\text { Aegle marmelos (L.) } \\
\text { Corr. Serr. }\end{array}$ & Rutaceae & Bela & $\mathrm{CF}$ & $\mathrm{E}$ & $\begin{array}{l}\text { Rt, Lf, } \\
\text { Fr }\end{array}$ & $\begin{array}{l}\text { Useful in diarrhea and dysentery, seminal } \\
\text { weakness, gastric, diabetes and asthmatic } \\
\text { complaints } \\
\end{array}$ \\
\hline $\begin{array}{l}\text { Ageratum conyzoides } \\
\text { L. } \\
\end{array}$ & Asteraceae & Poksungha & $\mathrm{CF}$ & E & $\mathrm{Rt}, \mathrm{Lf}$ & $\begin{array}{c}\begin{array}{l}\text { Used in cough, wound infection and eye } \\
\text { lotion. }\end{array} \\
\end{array}$ \\
\hline Albizia lebbek & Mimosaceae & Siris & $\mathrm{R}$ & $\mathrm{E}$ & $\begin{array}{l}\mathrm{Bk}, \mathrm{Fl}, \\
\mathrm{Sd}\end{array}$ & $\begin{array}{l}\text { Used in catarrh, asthma, leprosy, } \\
\text { leucoderma, sprain, wounds and ulcers. }\end{array}$ \\
\hline $\begin{array}{l}\text { Alstonia scholaris } \\
\text { (L.) R.Br }\end{array}$ & Apocynaceae & Chhatian & $\mathrm{R}$ & F H & $\mathrm{Bk}, \mathrm{Lf}$ & $\begin{array}{l}\text { Useful in preparation of cardio-tonic and } \\
\text { abdominal disorder, diarrhea, dysentery } \\
\text { and cardiopathy. }\end{array}$ \\
\hline $\begin{array}{c}\text { Andrographis } \\
\text { paniculata (Burm.f.) } \\
\text { Wall. ex Nees }\end{array}$ & Acanthaceae & Bhuinnimba & $\mathrm{CF}$ & E & Wp & $\begin{array}{l}\text { Wounds, ulcers, chronic fever, malaria, } \\
\text { skin diseases, and intestinal worms. }\end{array}$ \\
\hline
\end{tabular}




\begin{tabular}{|c|c|c|c|c|c|c|}
\hline $\begin{array}{c}\text { Anogeissus latifolia } \\
\text { (Roxb.) } \\
\text { Wall. ex Bedd. } \\
\end{array}$ & Combretaceae & Dhaura & $\mathrm{R}$ & E & $\begin{array}{l}\text { Rt, Bk, } \\
\text { Lf, Sd }\end{array}$ & $\begin{array}{l}\text { Haemostatic, useful in skin diseases, } \\
\text { diarrhea and dysentery. }\end{array}$ \\
\hline $\begin{array}{c}\text { Argemone mexicana } \\
\text { L. }\end{array}$ & Papaveraceae & Ganghauda & $\mathrm{R}$ & F H & $\mathrm{Wp}$ & $\begin{array}{c}\text { Antipyretic, sedative, aphrodisiac, used in } \\
\text { asthma, skin diseases, wounds and ulcers, } \\
\text { latex is used in jaundice and malaria } \\
\text { fever. }\end{array}$ \\
\hline $\begin{array}{l}\text { Asparagus } \\
\text { racemosus Willd. }\end{array}$ & Liliaceae & Satabari & $\mathrm{R}$ & E & Rt & $\begin{array}{c}\text { Useful in nervous disorder, diarrhea, } \\
\text { dysentery, throat infections, tuberculosis } \\
\text { and epilepsy, cardiac debility } \\
\text { hypertension and abortion. }\end{array}$ \\
\hline $\begin{array}{l}\text { Azadirachta indica } \\
\text { A. Juss. }\end{array}$ & Meliaceae & Neem & $\mathrm{R}$ & E & $\begin{array}{l}\text { Bk, Lf, } \\
\text { Sd, oil }\end{array}$ & $\begin{array}{l}\text { Useful in the cure of cough, skin diseases, } \\
\text { eczema, leucoderma, malaria, bronchitis } \\
\text { and diabetes. }\end{array}$ \\
\hline Bacopa monnieri & Scrophulariaceae & Brahmi & MQ & $\mathrm{V}$ & Wp & $\begin{array}{l}\text { Intellect promoting, anticonvulsant, } \\
\text { cardio tonic, useful in epilepsy and } \\
\text { insanity. }\end{array}$ \\
\hline $\begin{array}{c}\text { Bambusa } \\
\text { arundianacea }\end{array}$ & Bambusaceae & Kanta baunsa & $\mathrm{CF}$ & $\mathrm{S}$ & $\mathrm{Rt}, \mathrm{Lf}$ & $\begin{array}{c}\text { Skin diseases, ringworm, diarrhea, } \\
\text { gonorrhoea. }\end{array}$ \\
\hline $\begin{array}{l}\text { Bauhinia variegata } \\
\text { L. }\end{array}$ & Caesalpinaceae & Kanchana & $\mathrm{R}$ & E & Rt, Bk. & $\begin{array}{l}\text { Useful in skin diseases, leprosy, intestinal } \\
\text { worms, wounds and ulcers. }\end{array}$ \\
\hline Bombax ceiba $\mathrm{L}$. & Bombacaceae & Semul & $\mathrm{R}$ & E & $\begin{array}{l}\text { Rt, gum, } \\
\text { Bk, Fr. }\end{array}$ & $\begin{array}{l}\text { Paste is good for skin eruptions, fruits } \\
\text { useful in ulceration of bladder and kidney. }\end{array}$ \\
\hline $\begin{array}{l}\text { Buchanania lanzan } \\
\text { Spreng. }\end{array}$ & Anacardiaceae & Char & $\mathrm{CF}$ & $\mathrm{E}$ & $\mathrm{Rt}, \mathrm{Fr}$ & $\begin{array}{c}\text { Skin diseases, seminal weakness, cardio } \\
\text { tonic, nervous debility and cardiac } \\
\text { debility. }\end{array}$ \\
\hline $\begin{array}{l}\text { Calotropis gigantea } \\
\text { R.Br. }\end{array}$ & Asclepiadaceae & Arakha & MQ & F H & $\mathrm{Wp}$ & $\begin{array}{l}\text { Febrifuge and laxative. Promotes gastric } \\
\text { secretions. Useful for asthma, paralysis. }\end{array}$ \\
\hline $\begin{array}{l}\text { Careya arborea } \\
\text { Roxb. }\end{array}$ & Barriangtoniaceae & Kumbhi & $\mathrm{R}$ & E & $\begin{array}{l}\text { Bk, Lf, } \\
\text { Fr }\end{array}$ & $\begin{array}{l}\text { Thermogenic and antipyretic. Useful for } \\
\text { tumours. colic, intestinal worms, epileptic } \\
\text { fits and healing of vaginal ruptures. }\end{array}$ \\
\hline Cassia fistula $\mathrm{L}$. & Caesalpiniaceae & Sunari & $\mathrm{R}$ & E & $\mathrm{Wp}$ & $\begin{array}{c}\text { Useful in skin diseases, tuberculosis, } \\
\text { syphilis, diabetes, leprosy and ring worm. }\end{array}$ \\
\hline Cassia tora $\mathrm{L}$. & Caesalpiniaceae & Chakunda & $\mathrm{R}$ & F H & $\mathrm{Lf}, \mathrm{Sd}$ & $\begin{array}{l}\text { Thermogenic, laxative, liver tonic, cardio } \\
\text { tonic. }\end{array}$ \\
\hline Clitoria ternatea $\mathrm{L}$. & Fabaceae & Aparajita & $\mathrm{CF}$ & MH & $\begin{array}{l}\text { Rt, Lf, } \\
\text { Sd }\end{array}$ & $\begin{array}{c}\text { Useful in leprosy and leucoderma } \\
\text { treatment }\end{array}$ \\
\hline $\begin{array}{l}\text { Curcuma aromatica } \\
\text { Salisb }\end{array}$ & Zingiberaceae & Bana haladi & $\mathrm{CF}$ & $\mathrm{V}$ & $\mathrm{Rh}$ & $\begin{array}{l}\text { Useful in the treatment of bruises, sprains, } \\
\text { bronchitis, cough, leucoderma and skin } \\
\text { eruptions. }\end{array}$ \\
\hline Cuscuta reflexa & Convolvulaceae & Nirmuli & $\mathrm{R}$ & F H & $\mathrm{Wp}$ & $\begin{array}{c}\text { Useful in jaundice, cough, bronchitis and } \\
\text { paralysis. }\end{array}$ \\
\hline $\begin{array}{l}\text { Cynodon dactylon } \\
\text { (L.) Pers. }\end{array}$ & Poaceae & Duba ghassa & $\mathrm{CF}$ & E & $\mathrm{Wp}$ & $\begin{array}{l}\text { Haemostatic, useful in the treatment of } \\
\text { skin diseases, vomiting, diarrhea, } \\
\text { dysentery and abortion. }\end{array}$ \\
\hline Dalbergia sissoo & Fabaceae & Sissoo & $\mathrm{R}$ & $\mathrm{E}$ & $\begin{array}{l}\text { Rt, Lf, } \\
\mathrm{Bk}, \mathrm{Hw}\end{array}$ & $\begin{array}{l}\text { Thermogenic, aphrodisiac and } \\
\text { antipyretic. Useful in the treatment of skin } \\
\text { diseases, leucoderma, scabies, ulcers, } \\
\text { syphilis, gastropathy and } \\
\text { ophthalmopathy. }\end{array}$ \\
\hline Datura metel L. & Solanaceae & Dudura & $\mathrm{CF}$ & $\mathrm{FH}$ & $\mathrm{Wp}$ & $\begin{array}{l}\text { Narcotic, antispasmodic, intoxicant. } \\
\text { Useful in asthma, fever, ulcers and skin } \\
\text { diseases. Also used to treat dog bite. }\end{array}$ \\
\hline $\begin{array}{c}\text { Desmodium } \\
\text { gangeticum (L.) DC. }\end{array}$ & Fabaceae & Salaparni & $\mathrm{CF}$ & $\mathrm{E}$ & Rt & $\begin{array}{l}\text { Febrifuge, thermogenic, nervine tonic, } \\
\text { cardio tonic. }\end{array}$ \\
\hline $\begin{array}{l}\text { Emblica officinalis } \\
\text { Gaertn. }\end{array}$ & Euphorbiaceae & Anla & $\mathrm{CF}$ & $\mathrm{E}$ & $\begin{array}{l}\text { Rt, Bk, } \\
\text { Lf, Fr }\end{array}$ & $\begin{array}{l}\text { Aphrodisiac, antipyretic, digestive. } \\
\text { Useful for diabetes, asthma, peptic ulcers, } \\
\text { anaemia and emaciation. }\end{array}$ \\
\hline Ficus bengalensis $\mathrm{L}$. & Moraceae & Bara & $\mathrm{CF}$ & $\mathrm{E}$ & $\begin{array}{l}\text { Rt, Bk, } \\
\text { Lf, lt }\end{array}$ & $\begin{array}{l}\text { Useful in the treatment of diarrhea, } \\
\text { dysentery, diabetes, ulcers, skin diseases, } \\
\text { gonorrhoea, rheumatism. }\end{array}$ \\
\hline $\begin{array}{l}\text { Ficus microcarpa } \\
\text { L.f. }\end{array}$ & Moraceae & Jari & $\mathrm{R}$ & HT & $\begin{array}{l}\text { Rt, Bk, } \\
\text { Lf }\end{array}$ & $\begin{array}{l}\text { Useful in the treatment of wounds, ulcers, } \\
\text { diabetes, diarrhea and dysentery. }\end{array}$ \\
\hline Ficus racemosa $\mathrm{L}$. & Moraceae & Dimiri & $\mathrm{R}$ & HT & $\mathrm{Bk}, \mathrm{Fr}$ & $\begin{array}{l}\text { Useful in ulcers, leucoderma, anaemia, } \\
\text { jaundice and dysentery. }\end{array}$ \\
\hline Ficus religiosa $\mathrm{L}$. & Moraceae & Aswasthha & $\mathrm{R}$ & E & $\mathrm{Bk}, \mathrm{Fr}$, & Aphrodisiac, antibacterial and purgative. \\
\hline
\end{tabular}




\begin{tabular}{|c|c|c|c|c|c|c|}
\hline & & & & & $\mathrm{Sd}$ & Useful in skin infection. \\
\hline Flacourtia indica & Flacourtiaceae & Kanteikuli & $\mathrm{CF}$ & $\mathrm{E}$ & $\begin{array}{l}\text { Rt, Lf, } \\
\text { Fr }\end{array}$ & $\begin{array}{c}\text { Useful in the treatment snake bite, skin } \\
\text { diseases. Fruits are appetizer and } \\
\text { digestive. }\end{array}$ \\
\hline $\begin{array}{l}\text { Gardenia gummifera } \\
\text { L.f. } \\
\end{array}$ & Rubiaceae & Ghurudu & EX & HT & Resin & $\begin{array}{c}\text { Thermogenic, antispasmodic, } \\
\text { cardio-tonic. Useful in intestinal worms. }\end{array}$ \\
\hline $\begin{array}{l}\text { Gmelina arborea } \\
\text { Roxb. }\end{array}$ & Verbenaceae & Gambhari & $\mathrm{R}$ & HT & $\mathrm{Wp}$ & $\begin{array}{l}\text { Useful in fever, dyspepsia, skin diseases } \\
\text { and promoting the growth of hair. }\end{array}$ \\
\hline Helicteres isora $\mathrm{L}$. & Sterculiaceae & Mudmudi & $\mathrm{CF}$ & E & $\begin{array}{l}\text { Rt, Bk, } \\
\text { Fr }\end{array}$ & $\begin{array}{l}\text { Lactifuge, demulcent. Useful in colic, } \\
\text { scabies, diabetes and gastropathy. }\end{array}$ \\
\hline $\begin{array}{l}\text { Hemidesmus indicus } \\
\text { (L.) R.Br. }\end{array}$ & Asclepiadaceae & Anantamula & $\mathrm{CF}$ & $\mathrm{E}$ & $\begin{array}{l}\text { Rt, Lf, } \\
\text { St }\end{array}$ & $\begin{array}{c}\text { Aphrodisiac, demulcent, febrifuge, } \\
\text { Useful in asthma, bronchitis, leucoderma, } \\
\text { leprosy, epileptic fits. }\end{array}$ \\
\hline $\begin{array}{l}\text { Holarrhena } \\
\text { antidysenterica Wall } \\
\text { ex. A.DC. }\end{array}$ & Apocynaceae & Kurein & $\mathrm{CF}$ & $\mathrm{E}$ & $\begin{array}{l}\text { Bk, Sd, } \\
\quad \text { Lf }\end{array}$ & $\begin{array}{l}\text { Aphrodisiac, digestive and febrifuge. } \\
\text { Useful in amoebic dysentery, diarrhea, } \\
\text { asthma, skin diseases and chronic } \\
\text { bronchitis. }\end{array}$ \\
\hline $\begin{array}{c}\text { Ichnocarpus } \\
\text { frutescens (L.) R.Br. }\end{array}$ & Apocynaceae & Soyamlai & $\mathrm{CF}$ & $\mathrm{E}$ & Rt & $\begin{array}{l}\text { Seminal weakness, fever, skin diseases } \\
\text { and diabetes. }\end{array}$ \\
\hline $\begin{array}{l}\text { Indigofera cassioides } \\
\text { Rottl. ex DC. }\end{array}$ & Fabaceae & Nil & $\mathrm{R}$ & $\mathrm{E}$ & $\mathrm{Wp}$ & $\begin{array}{l}\text { Thermogenic, laxative. Useful for } \\
\text { promoting the growth of hair, cardiopathy } \\
\text { and neuropathy. }\end{array}$ \\
\hline $\begin{array}{l}\text { Lannea } \\
\text { coromondelica } \\
\text { (Houtt,) Merr. }\end{array}$ & Anacardiaceae & Mai & $\mathrm{CF}$ & $\mathrm{E}$ & $\mathrm{Bk}, \mathrm{Lf}$ & $\begin{array}{l}\text { Useful in treatment of wounds, bruises, } \\
\text { ulcers and sprains. }\end{array}$ \\
\hline $\begin{array}{l}\text { Lantana camara auct. } \\
\text { non L. }\end{array}$ & Verbenaceae & $\begin{array}{l}\text { Lantana (Naga } \\
\text { Airi) }\end{array}$ & $\mathrm{CF}$ & $\mathrm{FH}$ & $\mathrm{Wp}$ & $\begin{array}{c}\text { Antispasmodic. Useful in malaria, } \\
\text { epilepsy and gastropathy. }\end{array}$ \\
\hline $\begin{array}{l}\text { Leucas indica (L.) R. } \\
\text { Br. ex Vatke }\end{array}$ & Lamiaceae & Gayasha & $\mathrm{R}$ & $\mathrm{FH}$ & Lf, Fl & $\begin{array}{l}\text { Thermogenic, antipyretic. Leaf juice is } \\
\text { used as eye and nasal drop during } \\
\text { infection. }\end{array}$ \\
\hline $\begin{array}{l}\text { Limonia acidissima } \\
\text { L. }\end{array}$ & Rutaceae & Kainthha & MQ & HT & $\begin{array}{l}\text { Bk, Fr, } \\
\text { gum }\end{array}$ & $\begin{array}{l}\text { Cardio tonic.Useful in gastropathy, } \\
\text { diarrhea, vomiting, bronchitis. }\end{array}$ \\
\hline $\begin{array}{l}\text { Madhuca longifolia } \\
\text { (Koenig) Macbride }\end{array}$ & Sapotaceae & Mohul & $\mathrm{R}$ & $\mathrm{FH}$ & $\begin{array}{l}\mathrm{Bk}, \mathrm{Fr} \\
\mathrm{Sd}\end{array}$ & $\begin{array}{c}\text { Useful for epilepsy, sprains and } \\
\text { rheumatism. }\end{array}$ \\
\hline Mangifera indica $\mathrm{L}$. & Anacardiaceae & Amba & $\mathrm{R}$ & $\mathrm{FH}$ & $\begin{array}{l}\text { Rt, Bk, } \\
\text { Sd }\end{array}$ & $\begin{array}{l}\text { Useful for syphilis, wounds, ulcers, and } \\
\text { rheumatism. }\end{array}$ \\
\hline $\begin{array}{l}\text { Melia composite } \\
\text { Willd. }\end{array}$ & Meliaceae & Mohalimba & $\mathrm{R}$ & $\mathrm{S}$ & $\mathrm{Wp}$ & $\begin{array}{c}\text { Asthma, diabetes, fevers, after delivery, } \\
\text { hysteria, cardiac diseases and typhoid } \\
\text { fever. }\end{array}$ \\
\hline Mimosa pudica $\mathrm{L}$. & Mimosaceae & Lajkuli & $\mathrm{CF}$ & E & Rt, Lf & $\begin{array}{l}\text { Antispasmodic, Febrifuge, Useful in } \\
\text { leucoderma, jaundice, asthma, small pox } \\
\text { and uterus infection. }\end{array}$ \\
\hline $\begin{array}{l}\text { Morinda tinctoria } \\
\text { Roxb. non Noronha }\end{array}$ & Rubiaceae & Anchhu & $\mathrm{CF}$ & $\mathrm{E}$ & Rt, Lf & Useful in gastric disorder and diarrhea. \\
\hline $\begin{array}{l}\text { Mucuna pruriens (L.) } \\
\text { DC }\end{array}$ & Fabaceae & Baidanka & $\mathrm{CF}$ & $\mathrm{E}$ & $\begin{array}{l}\text { Rt, Lf, } \\
\text { Sd }\end{array}$ & $\begin{array}{l}\text { Thermogenic, stimulant, purgative. } \\
\text { Leaves are aphrodisiac. They are useful in } \\
\text { gonorrhoea and sterility. }\end{array}$ \\
\hline $\begin{array}{l}\text { Nyctanthes } \\
\text { arbortristis L. }\end{array}$ & Oleaceae & Gangasiuli & $\mathrm{CF}$ & $\mathrm{E}$ & $\begin{array}{l}\text { Lf, Fl, } \\
\quad \text { Sd }\end{array}$ & $\begin{array}{l}\text { Thermogenic, antibacterial and febrifuge. } \\
\text { Useful in malaria, chronic fever and } \\
\text { grayness of hair and baldness. }\end{array}$ \\
\hline $\begin{array}{l}\text { Ocimum americanum } \\
\text { L. }\end{array}$ & Lamiaceae & Banatulasi & $\mathrm{CF}$ & $\mathrm{E}$ & $\mathrm{Lf}, \mathrm{Sd}$ & $\begin{array}{c}\text { Useful in parasite infection and poisonous } \\
\text { bites, malarial fever. }\end{array}$ \\
\hline $\begin{array}{l}\text { Ougeinia oojeinensis } \\
\text { Roxb. }\end{array}$ & Fabaceae & Bandhan & EN & $\mathrm{V}$ & $\mathrm{Bk}$ & $\begin{array}{l}\text { Stimulant and rejuvenating. Useful in } \\
\text { diabetes, gonorrhoea and anaemia. }\end{array}$ \\
\hline $\begin{array}{l}\text { Pergularia daemia } \\
\text { (Forsk.) Chiov. }\end{array}$ & Asclepiadaceae & Uturudi & $\mathrm{R}$ & $\mathrm{F} \mathrm{H}$ & $\mathrm{Wp}$ & Useful in uterine and menstrual disorder. \\
\hline $\begin{array}{l}\text { Phoenix sylvestris } \\
\text { (L.) Roxb. }\end{array}$ & Arecaceae & Banakhajuri & $\mathrm{CF}$ & $\mathrm{FH}$ & Fr & $\begin{array}{l}\text { Cardio tonic and aphrodisiac. Useful in } \\
\text { seminal weakness and general debility. }\end{array}$ \\
\hline $\begin{array}{l}\text { Pterocarpus } \\
\text { marsupium Roxb. }\end{array}$ & Fabaceae & Piasal & $\mathrm{R}$ & $\mathrm{V}$ & $\begin{array}{l}\mathrm{Hw}, \\
\text { gum }\end{array}$ & $\begin{array}{l}\text { Rejuvenating and union promoter. Useful } \\
\text { in fractures, bruises, skin diseases, } \\
\text { leucoderma, diabetes. gum is used as liver } \\
\text { tonic. }\end{array}$ \\
\hline $\begin{array}{l}\text { Rauvolfia serpentina } \\
\text { (L.) Benth.ex Kurz. }\end{array}$ & Apocynaceae & Patal garuda & EN & $\mathrm{V}$ & Rt, Lf & $\begin{array}{c}\text { Useful for hypertension, insomnia, } \\
\text { epilepsy. }\end{array}$ \\
\hline Santalum album $\mathrm{L}$. & Santalaceae & Chandan & EN & $\mathrm{F} \mathrm{H}$ & $\mathrm{Hw}, \mathrm{Rt}$ & $\begin{array}{l}\text { Intellect promoting, cardio-tonic. } \\
\text { haemostatic, aphrodisiac. Useful in }\end{array}$ \\
\hline
\end{tabular}




\begin{tabular}{|c|c|c|c|c|c|c|}
\hline & & & & & & $\begin{array}{l}\text { psychopathy, cardiac debility, gastric } \\
\text { irritability and hyper acidity. }\end{array}$ \\
\hline $\begin{array}{l}\text { Schleichera oleosa } \\
\text { (Lour.) Oken }\end{array}$ & Sapindaceae & Kusum & $\mathrm{CF}$ & $\mathrm{E}$ & Bk, oil & $\begin{array}{c}\text { Bark used for malaria, ripening boils, seed } \\
\text { oil used for burns and ulcers. }\end{array}$ \\
\hline $\begin{array}{l}\text { Semecarpus } \\
\text { anacardium L.f. }\end{array}$ & Anacardiaceae & Bhalia & $\mathrm{R}$ & $\mathrm{E}$ & $\mathrm{Fr}$ & $\begin{array}{l}\text { Digestive, purgative, liver tonic stimulant, } \\
\text { antiseptic, uterine stimulant. Useful in } \\
\text { cancer, diabetes and tumor. }\end{array}$ \\
\hline $\begin{array}{l}\text { Sesbania grandiflora } \\
\text { (L.) Poiret }\end{array}$ & Fabaceae & Agasti & $\mathrm{R}$ & $\mathrm{S}$ & $\begin{array}{l}\text { Rt, Bk, } \\
\text { Lf, Fl, Fr }\end{array}$ & Febrifuge. Useful for nasal infection. \\
\hline $\begin{array}{l}\text { Shorea robusta } \\
\text { Gaertn.f. }\end{array}$ & Dipterocarpaceae & Sal & $\mathrm{R}$ & $\mathrm{FH}$ & $\mathrm{Bk}, \mathrm{Fr}$ & $\begin{array}{l}\text { Useful in bacterial infection, tuberculosis, } \\
\text { ulcers, Seminal weakness and burning of } \\
\text { eyes. }\end{array}$ \\
\hline $\begin{array}{l}\text { Solanum surattense } \\
\text { Burm. f. }\end{array}$ & Solanoceae & Ankaranti & $\mathrm{CF}$ & FH & Wp & $\begin{array}{l}\text { Digestive, febrifuge, stimulant, skin } \\
\text { diseases. hypertension, cough,asthma, } \\
\text { epilepsy, cardiac disorder. }\end{array}$ \\
\hline $\begin{array}{l}\text { Spondias pinnata } \\
\text { (L.f.) Kurz }\end{array}$ & Anacardiaceae & Ambada & $\mathrm{EN}$ & Above FH & $\begin{array}{l}\text { Rt, Bk, } \\
\text { Fr, Sd }\end{array}$ & $\begin{array}{l}\text { Thermogenic, appetizer and aphrodisiac. } \\
\text { Useful in muscular rheumatism. }\end{array}$ \\
\hline Streblus asper Lour. & Moraceae & Sahada & EN & $\mathrm{E}$ & $\begin{array}{l}\text { Rt, Bk, } \\
\text { Lf, latex }\end{array}$ & $\begin{array}{l}\text { Useful in cough, ulcers, bronchitis, throat } \\
\text { infection. }\end{array}$ \\
\hline $\begin{array}{l}\text { Syzygium cumini (L.) } \\
\text { Skeels }\end{array}$ & Myrtaceae & Jamu & $\mathrm{R}$ & E & $\begin{array}{l}\mathrm{Bk}, \mathrm{Lf}, \\
\mathrm{Fr}\end{array}$ & $\begin{array}{l}\text { Useful in diabetes and strengthening the } \\
\text { teeth, gums, ring worms. }\end{array}$ \\
\hline Tamarindus indica $\mathrm{L}$. & Caesalpiniaceae & Tentuli & $\mathrm{R}$ & $\mathrm{E}$ & $\begin{array}{l}\text { Rt, Fr, } \\
\text { Sd }\end{array}$ & $\begin{array}{c}\text { Thermogenic, anti-fungal, jaundice, } \\
\text { scabies, ringworm, small pox and } \\
\text { diabetes. }\end{array}$ \\
\hline Tectona grandis L.f. & Verbenaceae & Saguan & MQ & $\mathrm{FH}$ & Wp & $\begin{array}{l}\text { Useful in hyper acidity, diabetes, leprosy, } \\
\text { skin diseases. }\end{array}$ \\
\hline $\begin{array}{l}\text { Terminalia bellirica } \\
\text { (Gaertn.) Roxb. }\end{array}$ & Combretaceae & Bahada & $\mathrm{CF}$ & $\mathrm{E}$ & $\mathrm{Bk}, \mathrm{Fr}$ & $\begin{array}{c}\text { Useful in anaemia, leucoderma, narcotic, } \\
\text { digestive, antipyretic. }\end{array}$ \\
\hline $\begin{array}{l}\text { Terminalia chebula } \\
\text { Retz. }\end{array}$ & Combretaceae & Harida & $\mathrm{CF}$ & $\mathrm{E}$ & $\mathrm{Fr}$ & $\begin{array}{l}\text { Purgative, Digestive, Cardio tonic. Useful } \\
\text { in throat infection. }\end{array}$ \\
\hline $\begin{array}{l}\text { Terminalia } \\
\text { tomentosa Wight \& } \\
\text { Arn. }\end{array}$ & Combretaceae & Asan & $\mathrm{CF}$ & $\mathrm{E}$ & $\mathrm{Bk}$ & $\begin{array}{l}\text { Cardio tonic. Useful in throat infection } \\
\text { wounds, ulcers, diarrhea and dysentery. }\end{array}$ \\
\hline Tragia involucrate $\mathrm{L}$. & Euphorbiaceae & Bichhuati & $\mathrm{CF}$ & $\mathrm{E}$ & $\mathrm{Rt}, \mathrm{Lf}$ & $\begin{array}{c}\text { Venereal diseases, diabetes and blood } \\
\text { prifier. }\end{array}$ \\
\hline $\begin{array}{l}\text { Trichosanthes } \\
\text { anguina }\end{array}$ & Cucurbitaceae & Mohakal & $\mathrm{R}$ & $\mathrm{E}$ & $\mathrm{Rt}, \mathrm{Fr}$ & $\begin{array}{l}\text { Useful in asthma, ophthalmia epilepsy } \\
\text { and leprosy. }\end{array}$ \\
\hline Vitex negundo L. & Verbenaceae & Begunia & $\mathrm{R}$ & F H & $\mathrm{Wp}$ & $\begin{array}{l}\text { Antiseptic, antipyretic, ophthalmic, } \\
\text { general debility, sprains and cardiac } \\
\text { disorder. }\end{array}$ \\
\hline $\begin{array}{l}\text { Vitis quadrangularis } \\
\text { Wall. ex Lawson }\end{array}$ & Vitaceae & Hadabh-anga & MQ & $\mathrm{E}$ & $\mathrm{Wp}$ & $\begin{array}{c}\text { Used in treatment of fractures, swellings, } \\
\text { powdered roots and stem paste are used in } \\
\text { specifically for bone fractures. }\end{array}$ \\
\hline $\begin{array}{l}\text { Woodfordia fruticosa } \\
\text { (L.) Kurz. }\end{array}$ & Lythraceae & Dhatki & $\mathrm{CF}$ & $\mathrm{E}$ & $\mathrm{Fl}$ & $\begin{array}{l}\text { Stimulant, antibacterial corrective of } \\
\text { urinary pigments, and diabetes. }\end{array}$ \\
\hline $\begin{array}{l}\text { Ziziphus mauritiana } \\
\text { Lam. }\end{array}$ & Rhamnaceae & Barakoli & $\mathrm{CF}$ & $\mathrm{E}$ & $\mathrm{Wp}$ & $\begin{array}{l}\text { Aphrodisiac and invigorating. Useful in } \\
\text { the treatment of boils, wounds, syphilis, } \\
\text { ulcers, typhoid fever.. }\end{array}$ \\
\hline
\end{tabular}

Note: $\mathrm{CF}=$ commonly found, $\mathrm{R}=$ Rare, $\mathrm{MQ}=$ Medium quantity, $\mathrm{EN}=$ Endangered, $\mathrm{EX}=$ Extinct, $\mathrm{S}=$ Slope areas, $\mathrm{MH}=$ Middle of Hills, $\mathrm{V}=\mathrm{Valley}, \mathrm{HT}=\mathrm{Hill}$ Top, FH=Foot Hill, E=Every where, Fl-flower; Fr-fruit; Lf-leaf; Bk-bark; Rt-root; Rh-rhizome; St-stem; Sd-seeds; Wp-whole plant

Local users rely on subsistence agriculture; with plants performing vital roles as crops, fodders, fruits and vegetables, fuel wood, building materials and medicines. Moreover, some plant species have religious value; some are used for construction as timber, while many of them are used for edible purpose. Local users use plant parts such as bark, root, leaf, seed, flower, fruit or entire plant for medicinal purpose to cure their different nature of ailments. People rely on plant-based medicines for primary health care against stomachache, diarrhea, bacillary dysentery, colic dysentery, constipation, digestion problem, gastric, stomach pain, rheumatic problems, respiratory problems such as cough, cold, bronchitis, asthma, tuberculosis, fever, skin diseases and bone fracture.

\section{Discussion}

Plants are of utmost interest to the human race and our ancestors lived on nuts, roots, succulent stems, fruits and other parts of plants. Today, our existence can still not be imagined without plants. The Mandaragiri forest support a number of plant species, most of which have useful values. Local people are based on agriculture supported by animal 
husbandry and dependent upon the forest resources around them for a number of daily requirements such as fodder, fuel wood, medicine, food, and fiber, raw material for handicrafts, house construction and agricultural tools. Most of the identified plants reported here have multiple local uses. Some commercially important plant species such as Anogeissus latifolia, Gmelina arborea, Madhuca longifolia, Pterocarpus marsupium, Shorea robusta, Terminalia arjuna and Terminalia bellirica have also medicinal value. Some wild food plants viz Buchanania, Flacourtia, Spondias etc. have medicinal properties and in other parts of the country are well known for their edible.

Aegle marmelos, Ocimmum americanum,Achyranthes aspera, Calotropis gigantea, Ficus bengalensis, Emblica officinalis. Semecarpus anacardium and Mangifera indica are regarded for religious purpose along with their medicinal value and other domestic uses, and found to be well conserved by the tribal people.

Considering that the demand for medicinal plants is increasing, their survival in their natural habitat is under growing threat. This is due to over-harvesting, rapid degradation and loss of natural habitats. Thirty five medicinal plant species are critically threatened in Odisha state. Species such as Acacia catechu, Acacia nilotica, Buchanania lanzan, Emblica officinalis, Gardenia sp., Indigofera cassioides, Shorea robusta and Woodfordia fruticosa are rare, while, plants like Rauvolfia serpentina, Santalum album, Streblus asper and Spondias pinnata are endangered species at Mandaragiri forest.

Today, it is estimated that about 1,000 species of medicinal plants are facing threat to their existence, and some of them have become extinct in the wild. The Mandragiri forest constitutes a vast treasure of diverse medicinal plants. Many of the identified medicinal species and many more are yet to be identified may exist from Mandragiri. Therefore, steps must be taken for their identification and conservation both by ex-situ and in-situ means.In order to protect the biological diversity following measures are advise for conservation purpose viz. (i) In-situ conservation of wild population in forests and other in-situ habitats like sacred grooves and community forests; (ii) Establishment of herbal gardens to conserve representative populations of medicinal plants (iii) Establishment of several home gardens to conserve intra-specific diversity of plants useful for human and livestock health; (iv) Large scale organic cultivation of selected cultivars of medicinal plants. Many authors have advocated the involvement of local people in the protection of natural resources, such as forests and wildlife. So, involvement of local peoples can also play a major role in the conservation with following ways- (i) Generate and share information regarding species of medicinal and economic importance and conservation concern; (ii) Promote awareness of the concerns and policies tribes and other groups that safe guard traditional knowledge of native medicinal plants as these pertain to confidentially of information; (iii) Encourage active participation by tribes and other holders of traditional ecological knowledge pertaining to native medicinal plants; and (iv) Conserve indigenous plants and plant communities used in traditional medicines, ceremony, ethnobotany and the natural products industry.

\section{Conclusion}

The present study indicates that Mandaragiri forest harbors a high diversity of useful plants. The inhabitants are dependent on folk medicine due to poverty and the traditional belief of its effectiveness. Documentation of availability of medicinal plants has provided useful information. Further ethno-medicinal studies are required from this area, as tribes have a strong belief in the efficacy and success of herbal medicine and traditional healing practices among them. Therefore, it is essential to conserve such a wealth of information hidden among the local people.

\section{Acknowledgements}

Authors are grateful to the Mr. Pandab Behera, IFS, RCCF, Angul and Vinod Kumar, IFS, PD, OFSD for allowing permission for this study. Thanks are due to forest staff and tribal who cooperated in sharing their knowledge during the study.

\section{REFERENCES}

[1] R Govaerts. How many species of seed plants are there? Taxon, vol. 50(4), 1085-1090, 2001.

[2] PH Raven. Medicinal plants and global sustainability: The canary in the coal mine. In Medicinal Plants: A Global Heritage, Proceedings of the International conference on medicinal plants for survival, New Delhi: International Development Research Center, 14-18, 1998.

[3] P Pushpangadan. Ethnobiology of India: A Status Report, GOI, New Delhi, 1995.

[4] SK Ghimire, D McKey, Y Aumeeruddy-Thomas. Heterogeneity in ethnoecological knowledge and management of medicinal plants in the Himalayas of Nepal: implications for conservation. Ecology and Society, vol. 9(3): 6, 2004.

[5] TB Henfrey. Ethnoecology, Resource Use, Conservation and Development in a Wapishana Community in the South Rupununi Guyana. (PhD thesis) University of Kent, UK, 2002.

[6] T Ticktin, T Johns. Chinanteco management of Aechmea magdalenae: implications for the use of traditional ecological knowledge and traditional resource management in management plans. Economic Botany, vol. 56(2), 117-191, 2002.

[7] M Gadgil, F Berkes, C Folke-. Indigenous knowledge of biodiversity conservation. Ambio, vol. 22(2-3), 151-156, 
1993.

[8] RM Kunwar, N Adhikari. Ethnomedicine of Dolpa district, Nepal: the plants, their vernacular names and uses. Lyonia, vol. 8(1), 41-47, 2005.

[9] CP Kala. Current status of medicinal plants used by traditional Vaidyas in Uttaranchal- State of India. Ethnobotany Research and Applications, 3, 267-278, 2005.

[10] HH Haines. The Botany of Bihar and Orissa. London. (Reprint, 1961, Calcutta), 1921-1925.

[11] KR Kirtikar, BD Basu, ICG An. Indian Medicinal Plants, Vol.
II. Edited \& rewritten by Blatter E, Caius JF \& Mhaskar KS. $2^{\text {nd }}$ edition, (International Book Distributors, Dehradun, India), 2006.

[12] KR Kirtikar, BD Basu, ICG An. Indian Medicinal Plants, Vol. I. Edited \& rewritten by Blatter E, Caius JF \& Mhaskar KS. $2^{\text {nd }}$ edition, (International Book Distributors, Dehradun, India), 2005.

[13] KR Kirtikar, BD Basu, ICG An. Indian Medicinal Plants, Vol. IV. Edited \& rewritten by Blatter E, Caius JF \& Mhaskar KS. $2^{\text {nd }}$ edition, (International Book Distributors, Dehradun, India), 2007. 